\section{Rund ums}

\section{Lungenkarzinom}

Tabakassoziierter Lungenkrebs ist bei Männern die häufigste Krebstodesursache, so die ersten Ergebnisse eines Gesundheitsberichts des Robert-KochInstituts.

Eine Datenauswertung von 722 Patienten mit NSCLC zeigte, dass Betablocker die Gesamtmortalität um 22\% während eines mittleren Follow-Ups von 44 Monaten senken können.

Laut einer schwedischen Analyse liegt das absolute Risiko, bis zum 75. Lebensjahr an Lungenkrebs zu erkranken, bei familiärer Vorbelastung bei $5 \%$.

\section{Obacht: Fast Food macht Asthma!}

Das von Kindern und Jugendlichen so geliebte Fast-Food steht im Zusammenhang mit Asthma, Heuschnupfen oder Ekzemen. Dies legt zumindest die bisher weltweit größte epidemiologische Studie ISAAC (International Study of Asthma and Allergies in Childhood) nahe. Kinder und Jugendliche, die mindestens dreimal pro Woche Burger, Pommes und Co. essen, leiden vergleichsweise häufig an schweren Symptomen. Das Risiko schwerer Asthmasymptome stieg bei Jugendlichen um 39\%. Steht hingegen viel Obst auf dem Speiseplan, erkranken die Heranwachsenden seltener.

Ellwood P et al, Thorax 2013 (online first)

\section{Wer am Steuer in den Seilen hängt...}

... leidet möglicherweise an obstruktiver Schlafapnoe und erhöht damit das Risko für Verkehrsunfälle um das Achtfache! Berufsfahrer müssen trotzdem nicht um ihren Job bangen, denn mit konsequenter CPAP sinken Unfallgefahr und Behandlungszeit in der Klinik drastisch. Dies und vieles mehr lesen Sie in unserem Schwerpunkt "Atemwege“.

Claudia Daniels Redakteurin

\section{Bei N2-Lungenkrebs bringt Bestrahlen keinen Vorteil}

Viele Patienten, die an einem nicht-kleinzelligen Bronchialkarzinom (NSCLC) im Stadium III leiden, werden nach kompletter Resektion des Tumors noch einer Strahlentherapie unterzogen. Laut einer aktuellen US-Studie verbessert dies die Überlebenschancen jedoch nicht. In der retrospektiven Studie werteten Juan Wisnievesky und Kollegen Daten von $1307 \mathrm{~Pa}-$ tienten aus, die an einem NSCLC mit Beteiligung der ipsilateralen, mediastinalen oder subkarinalen Lymphknoten (N2) er- krankt waren. Die Überlebensraten der postoperativ bestrahlten und der nicht bestrahlten Patienten waren statistisch betrachtet nach einem und nach drei Jahren gleich. „Dieses Ergebnis sollte erhebliche Konsequenzen für die Klinik haben“, fordern die Wissenschaftler. Denn die Daten zeigten, dass trotz fehlender Wirkungsnachweise ein großer Prozentsatz dieser Patienten eine Strahlentherapie erhalte.

Wisnivesky JP et al, Cancer 2012; DOl: 10.1002/cncr.26585

\title{
Besser seitlich als Heimlich?
}

Druck von vorne, von hinten oder von der Seite - es gibt verschiedene Techniken, die Atemwege von aspirierten Fremdkörpern zu befreien. Doch welche Methode ist die beste? Laut den Messungen australischer Wissenschaftler lässt sich zwar mit dem bekannten HeimlichManöver zum Aufbau abdominellen Drucks der höchste Druckwert erzielen $\left(142,4 \mathrm{cmH}_{2} \mathrm{O}\right)$. Davon kommen jedoch nur maximal
$13,8 \mathrm{cmH}_{2} \mathrm{O}$ in den Atemwegen an. Pressmanöver auf die laterale Thoraxwand erzielen zwar nicht so hohe Drücke, der Spitzendruck in den Atemwegen steigt aber auf $18,0 \mathrm{cmH}_{2} \mathrm{O}$. Damit weist das laterale Pressen eine höhere Effizienz auf als der Heimlich-Handgriff. Auch das Risiko für Organverletzungen ist geringer. Hier muss allerdings erwähnt werden, dass es sich bei den „Probanden“ der Studie um Hausschweine handelte. Bevor sich an den Regeln, wie bei Fremdkörperaspiration vorzugehen sei, etwas ändern kann, sind noch weitere Studien am Humanmodell nötig.

Lippmann J et al, Resuscitation 2012 (online first)

\section{So lange darf ein Husten dauern}

Viele Patienten unterschätzen die normale Dauer eines Erkältungshustens. Ärzte um Dr. Mark Ebell von der University of Georgia haben die durchschnittliche Dauer eines akuten Hustens bei unspezifischer Atemwegsinfektion in einer Metaanalyse, für die 19 Studien mit 23 bis 1230 Patienten herangezogen wurden, ermittelt und mit der Erwartungshaltung von Patienten verglichen. Bis ein Erkältungshusten sich vollständig legt, vergehen bei ansonsten gesunden Erwachsenen üblicherweise zwei bis drei Wochen. Die meisten Patienten glauben jedoch, dass der Husten schon nach fünf bis sieben Tagen verschwunden sein müsste. Ebell und Kollegen vermuten, dass diese Fehleinschätzungen zu unnötigen Antibiotikaverordnungen führen können. Um dies zu verhindern, sollte man die Patienten darüber aufklären, dass einem Erkältungshusten in den meisten Fällen ein viraler Infekt zugrunde liegt. Ebenso sei es wichtig, $\mathrm{Pa}-$ tienten den regulären Verlauf eines akuten Hustens klar zu machen. Ein Arztbesuch ist laut Ebell nur dann notwendig, wenn eine Verschlechterung eintritt oder wenn Alarmsymptome wie hohes Fieber, blutiges Sputum oder Kurzatmigkeit hinzukommen.

Ebell MH et al, Ann Fam Med 2013, 11:5-13 